\title{
Blepharhymenus koreanusPaśnik and Tachyusa wei Pace New to South Korea (Coleoptera: Staphylinidae: Aleocharinae)
}

\author{
Jeong-Hun Song and Kee-Jeong Ahn* \\ Department of Biology, Chungnam National University, Daejeon 305-764, Republic of Korea
}

\section{님한 미기록종 호리바수염반날개와 배구멍바수염반널개}

\author{
송정훈 · 안기정* \\ 대전 충남대학교 생물과학과
}

\begin{abstract}
Blepharhymenus koreanus Paśnik and Tachyusa wei Pace are recorded for the first time in South Korea. Habitus photographs, redescriptions and illustrations of diagnostic characters are provided.
\end{abstract}

Key words: Coleoptera, Aleocharinae, Blepharhymenus, Tachyusa, New record

초 록: 남한 미기록종인 Blepharhymenus koreanus Pasnik (호리바수염반날개)(신칭)과 Tachyusa wei Pace (배구멍바수염반날개)(신칭)을 처음으 로 기록한다. 두 종에 대한 성충의 사진, 재기재 및 주요 형질에 대한 그림을 함께 제시한다.

검색어: 딱정벌레목, 바수염반날개아과, 호리바수염반날개, 배구멍바수염반날개, 미기록종

The Aleocharinae have notoriously been known as the most taxonomically difficult group (Arnett, 1968). In the Korean Peninsula, 188 species in 63 genera are recorded at present (Ahn et al., unpublished observation). For ongoing study of aleocharine diversity in Korea, we have identified two oxypodine species (Blepharhymenus koreanus Paśnik and Tachyusa wei Pace) new to South Korea.

Paśnik (2001) first described B. koreanus including many other aleocharine beetles from North Korea and revised the genus Tachyusa Erichson including 53 species with $T$. wei (2006). However, these two species were reported without sufficient illustrations of diagnostic characters and detailed descriptions. In this paper we provide illustrations of habitus, redescriptions, and line drawings of diagnostic characters. The

*Comesponding author: kjahn@cnu.ac.kr

Received June 21 2013; Revised September 22013

Accepted September 72013 specimens studied are deposited in the Chungnam National University Insect Collection (CNUIC), Daejeon, Korea.

\section{Taxonomic accounts}

\section{Blepharhymenus koreanus Paśnik, 2001 호리바수염반날개} (신칭)

(Figs. 1A, 2)

Blepharrhymenus koreanus Paśnik, 2001: 227.

Blepharhymenus koreanus: Smetana, 2004: 466.

Redescription. Length about 2.8-3.0 mm. Body (Fig. 1a) reddish brown to dark brown, abdominal tergites IV-VI darker. Head (Fig. 2a) circular, with narrow neck, head and pronotum subequal in width. Antennae long, antennomere 2 slightly longer than 1, 3 shorter than 2, 4 elongate, 5-6 about as long as wide, $7-10$ weakly transverse, 11 longest, longer than preceding 


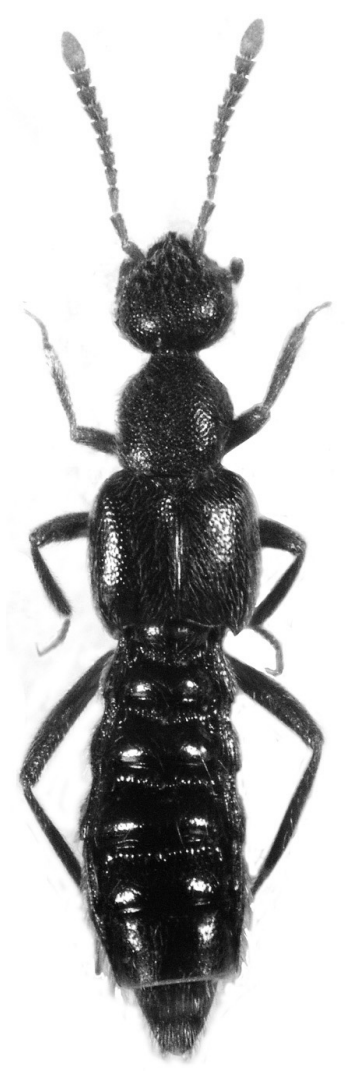

(a)

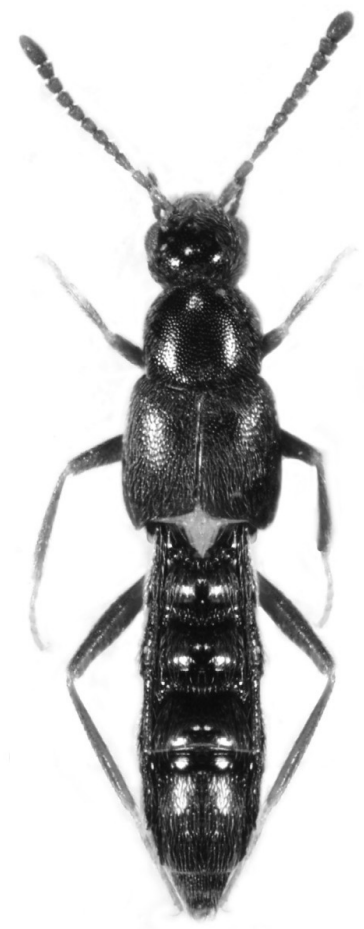

(b)
Fig. 1. Habitus. (a) Blepharhymenus koreanus, $3.0 \mathrm{~mm}$; (b) Tachyusa wei, $2.5 \mathrm{~mm}$.

two combined. Labrum (Fig. 2b) transverse, prominent anteriorly at middle in anterior margin, 2 lateral sensilla and about 26-27 macrosetae present on each side of midline. Labium (Fig. 2c) with ligula not divided, slender, elongate, longer than labial palpomere 1; medial setae close to each other; median pseudopores, lateral pseudopores, 2 setal pore and 4 real pores present on prementum; labial palpus elongate; palpomere 1 shortest, 2 about 1.4 times longer than 1, 3 longest, slightly longer than 2, slender, about 4.6 times longer than wide. Pronotum (Fig. $2 \mathrm{~d}$ ) bottle gourd shaped, approximately 1.2 times as long as wide, widest at apical $2 / 5$; densely punctate, coarsely pubescence. Elytron 1.8 times longer than wide, postero-lateral margin sinuated, outer margin arcuated. Mesoventral process elongate and weakly rounded at apex, much longer than metaventral process, metaventral process broader than mesoventral process, weakly rounded at apex; length ratio of mesoventral process, isthmus and metaventral process 7:1.3:2.4.
Tergites III-VI with deep transverse impression with punctures and medial carina. Median lobes as in Figs. 2e-f.

Materials examined. Total 43 specimens. KOREA: Chungnam Prov., Nonsan-si, Beolgok-myeon, Surak-ri, Surak-valley,

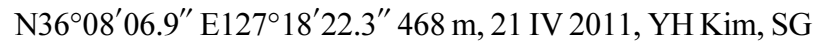
Lee, near stream; 7 ex (in alcohol), N36 $07^{\prime} 48.8^{\prime \prime}$ E127 $18^{\prime}$ $30.2^{\prime \prime} 433$ m, 21 IV 2011, YH Kim, SG Lee, leaf litter near stream; 2 ex (in alcohol), N36 $08^{\prime} 06.9^{\prime \prime}$ E127 $18^{\prime} 22.3^{\prime \prime} 468 \mathrm{~m}$, 21 IV 2011, YH Kim, SG Lee, leaf litter near stream; Gangwon Prov., 1 ex, Taebaek-city, Mt. Taebaeksan, Baekdansa, 14 VII 1999, US Hwang, ex sifting; Gyeongbuk Prov., 1 ex Mungyeongseje, 8 V 1999, YB Cho; 5 ex (20¹ 1 으, on slides) Tangyang-gun, Mt Sobaeksan, Chôndong Area, 8-9 V 1999, US Hwang, HJ Kim, ex sifting; 1 ex, Tangyang-gun, Mt Sobaeksan, Chôndong Area, 8-9 V 1999, US Hwang, HJ Kim, ex sifting; Gyeongnam Prov., 1 ex, Hamyang-gun, Macheon, Mt Jirisan, Baekmudong, 18 VII 2002, YB Cho, ex sifting; 22 ex (in alcohol), Macheon-myeon, Mt Jirisan, Hongdae-fall, $\mathrm{N} 35^{\circ} 20^{\prime} 44.2^{\prime \prime} \mathrm{E} 127^{\circ} 40^{\prime} 59.7^{\prime \prime} 740$ m, 15 VI 2010, KJ Ahn, TK Kim, JG Lee, IS Yoo, JH Song, SG Lee; 3 ex (in alcohol), Yongso-valley, N35²3'03.4"E1274' $53.2^{\prime \prime} 410$ m, 14 VI 2010, KJ Ahn, TK Kim, JG Lee, IS Yoo, JH Song, SG Lee; 4 ex (in alcohol).

Distribution. North and South Korea.

Remarks. Paśnik (2001) used "Blepharrhymenus" but Newton and Thayer (1992: 53) corrected it as "Blepharhymenus." See (Bouchard et al., 2011: 204) for detailed comments. Blepharhymenus koreanus is similar to $B$. chinensis Bernhauer, but can be distinguished by the antennomere 3 shorter than the antennomere 2 , the coarser punctuation of head and elytra, the shorter elytra, and the bases of tergites III-VI with transverse impression (Paśnik, 2001: 228).

\section{Tachyusa wei Pace, 1993 배구멍바수염반날개(신칭)}

(Figs. 1B, 3)

Tachyusa (Caliusa) wei Pace, 1993: 88; Paśnik, 2006: 103; Paśnik, 2010: 623.

Ischnopoda (Caliusa) hebeiensis Pace, 1998: 404. (Synonymized by Paśnik, 2006)

Tachyusa coarctata: Paśnik, 2001: 194. (Misidentification)

Ischnopoda hebeiensis: Smetana, 2004: 490. (as valid species)

Ischnopoda wei: Smetana, 2004: 490. (as valid species) 


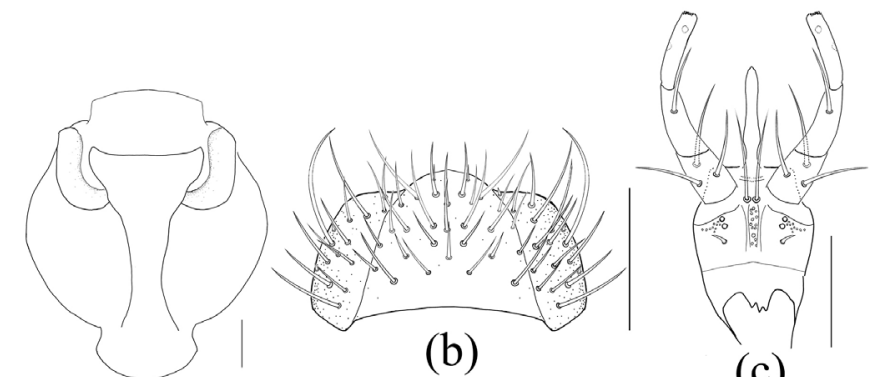

(a)

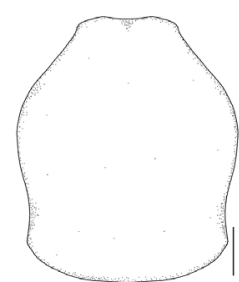

(d)

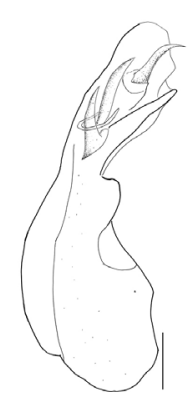

(e) (c)

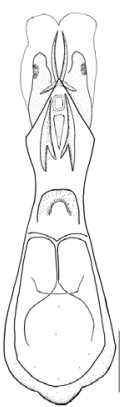

(f)

Fig. 2. Blepharhymenus koreanus. (a) head, ventral aspect; (b) labrum, dorsal aspect; (c) labium, ventral aspect; (d) pronotum, dorsal aspect; (e) median lobe, lateral aspect; $(\mathrm{f}$ ) median lobe, ventral aspect. Scales $=0.1 \mathrm{~mm}$.

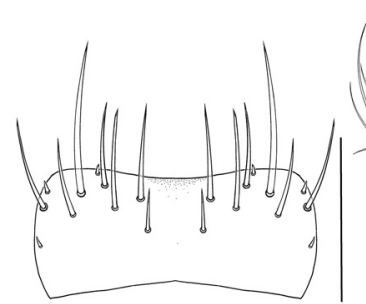

(a)

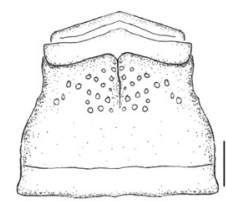

(d)

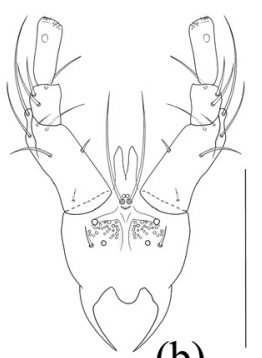

(b)

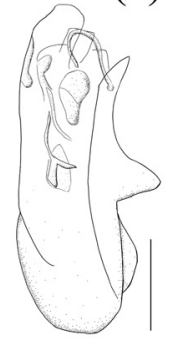

(e)

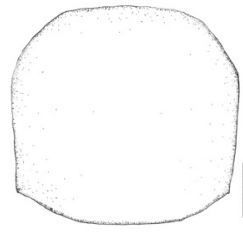

(c)

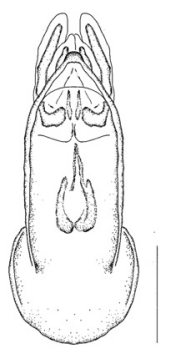

(f)

Fig. 3. Tachyusa wei. (a) labrum, dorsal aspect; (b) labium, ventral aspect; (c) pronotum, dorsal aspect; (d) sternite III, ventral aspect; (e) median lobe, lateral aspect; $(\mathrm{f})$ median lobe, ventral aspect. Scales $=0.1 \mathrm{~mm}$.

Redescription. Length 2.5-3.2 mm. Body (Fig. 1b) slender, glossy, pitchy brown to black, tergites III-V often paler, brownish red. Legs testaceous with tarsi yellow. Antennae brown with antennomeres 1-3 reddish yellow. Head, convex, puncture fine and dense, eyes large, weakly prominent, about as long as tempora. Antennae long and slender, antennomere 1about as long as 2, 3 slightly longer than 2, 4-6 elongate, 7-8 approximately as long as wide, 9-10 weakly transverse, 11 longest, slightly shorter than preceding two combined. Labrum (Fig. 3a) transverse, slightly emarginate in anterior margin, 2 
lateral sensilla and about 7 macrosetae present on each side of midline. Labium (Fig. 3b) with ligula divided into 2 lobes in basal half; medial setae close to each other; two basal pores close together; lateral pseudopores, 2 setal pore and 4 real pores present on prementum; labial palpus elongate, with many setulae; palpomere 1 largest, about 2.3 times longer than 2, 2 shortest, 3 about 1.6 times longer than 2. Pronotum (Fig. 3c) quadrate, about as long as wide, densely and finely punctate, pubescence at midline directed posteriorly. Elytron 1.64 times longer than wide, postero-lateral margin weakly sinuated, outer margin arcuated. Meso- and metaventral processes elongate and weakly rounded at apex, mesoventral process slightly longer than metaventral process. Tergites III-V with a deep transverse impression, with punctures, densely, small and well visible. Tergite III longer than wide, posterior margin of tergite IX straight. Sternites III-V (Fig. 3d, sternite III) with medial carina at middle. Median lobes as in Figs. $3 \mathrm{e}^{-\mathrm{f}}$.

Materials examined. Total 16 specimens. KOREA: Chungnam Prov., 12 ex (20", on slides), Daejeon-city, Gasuwon-dong, Gapcheon stream, 9 VI 1999, HJ Kim, ex near stream; 14 ex (1 오, on slides), US Hwang, near stream; 3 ex (1 $0^{7}$, on slides), Heukseok-dong, 25 VI 1999, HJ Kim, ex near stream; 6 ex, Yuseong-gu, Chungnam-univ, 13 VI 2003, SM Choi, JS Park, near stream; Gangwon Prov., 3 ex, Donghae-city, Mureunggyegok, 5 VII 1991, KJ Ahn, near stream; Wonju-city, Munmak-eup, Geondong reservoir, 25 v 2002, CW Shin, JS Park, ex near stream; Gyeonggi Prov., Pocheon-gun, Sanjeong lake, 22 VI 2003, JS Park, N38 $07^{\prime} 101^{\prime \prime}$ E127 $32^{\prime} 32.7^{\prime \prime}$, ex near stream; Jeonbuk Prov., 21 ex (1 $10^{\top} 2$ 우, on slides), Jeongeup-city, Mt. Naejangsan, Paekyang area, 25-26 V 1999, US Hwang, HJ Kim, FIT.

Distribution. North and South Korea, China and Japan.

Remarks. Paśnik (2001) first reported Tachyusa coarctata from North Korea, but it was an incorrect identification of Tachyusa wei (Paśnik, 2006: 96). Tachyusa wei is extremely similar to T.orientis Bernhauer 1938, but can be distinguished by tergite III longer than wide, sternite III-V without medial carina, and different shape of the median lobe (Figs. $3 \mathrm{e}^{-} \mathrm{f}$ ).

\section{Acknowledgements}

We thank Dr. Grzegorz Paśnik (Institute of Systematics and Evolution of Animals, ISEA) for his suggestions on the identification of Korean oxypodine species. This work was supported by a grant from the National Institute of Biological Resources (NIBR), funded by the Ministry of Environment (MOE) of the Republic of Korea (NIBR No. 2013-02-001).

\section{Literature Cited}

Arnett, R.H. Jr., 1968. The Beetles of the United States (A Manual for Identification). Catholic University Press, Washington, DC. $1112 \mathrm{pp}$.

Bouchard, P., Bousquet, Y., Davies, A.E., Alonso-Zarazaga, M.A., Lawrence, J.F., Lyal, C.H.C., Newton, A. F., Reid, C.A.M., Schmitt, M., Ślipiński, S.A., Smith, A.B.T., 2011. Family-group names in Coleoptera (Insecta). ZooKeys. 88, 1-972

Newton, A.F., Thayer, M.K., 1992. Current classification and family-group names in Staphyliniformia (Coleoptera). Fieldiana.

Pace, R., 1993. Aleocharinae della Cina (Coleoptera, taphylinidae). Boll. Mus. civ. St. nat. Verona, 17, 69-125.

Pace, R., 1998. Aleocharinae della Cina: Parte II (Coleoptera, taphylinidae). Revue suisse de Zoologie. 105(2), 395-463.

Paśnik, G., 2001. The North Korean Aleocharinae (Coleoptera, Staphylinidae): diversity and biogeography. Acta Zoological Cracoviensia. 44, 185-234.

Paśnik, G., 2006. A revision of the World species of the genus Tachyusa Erichson, 1837 (Coleoptera, Staphylinidae: leocharinae). Zootaxa. 1146, 1-152.

Paśnik, G., 2010. The Tachyusa coarctata species group revisited: Phylogenetic relationships and a new species (Coleoptera: Staphylinidae: Aleocharinae). Eur. J. Entomol. 107(4), 621-629. Smetana, A., 2004. Subfamily Aleocharinae Fleming, 1821. In: Löbl, I., Smetana, A. (Eds.), Catalogue of Palaearctic Coleoptera, Volume. 2 Hydrophiloidea-Histeroidea-Staphylinoidea, Apollo Books, Stenstrup, pp. 353-494. 